\title{
Endotracheopulmonary Instillation, Powder and Solvent for Suspension Dosage Form
}

National Cancer Institute

\section{Source}

National Cancer Institute. Endotracheopulmonary Instillation, Powder and Solvent for

Suspension Dosage Form. NCI Thesaurus. Code C149489.

Powder and aqueous solvent intended for the preparation of an endotracheopulmonary instillation suspension by dispersing the powder in the solvent. 